\title{
Quantitative multi-parametric evaluation of centrosome declustering drugs: centrosome amplification, mitotic phenotype, cell cycle and death
}

\author{
A Ogden ${ }^{1}$, A Cheng ${ }^{1}$, PCG Rida ${ }^{1}$, V Pannu ${ }^{1}$, R Osan ${ }^{2}$, R Clewley ${ }^{3}$ and R Aneja ${ }^{*, 1}$
}

Unlike normal cells, cancer cells contain amplified centrosomes and rely on centrosome clustering mechanisms to form a pseudobipolar spindle that circumvents potentially fatal spindle multipolarity (MP). Centrosome clustering also promotes lowgrade chromosome missegregation, which can drive malignant transformation and tumor progression. Putative 'centrosome declustering drugs' represent a cancer cell-specific class of chemotherapeutics that produces a common phenotype of centrosome declustering and spindle MP. However, differences between individual agents in terms of efficacy and phenotypic nuances remain unexplored. Herein, we have developed a conceptual framework for the quantitative evaluation of centrosome declustering drugs by investigating their impact on centrosomes, clustering, spindle polarity, cell cycle arrest, and death in various cancer cell lines at multiple drug concentrations over time. Surprisingly, all centrosome declustering drugs evaluated in our study were also centrosome-amplifying drugs to varying extents. Notably, all declustering drugs induced spindle MP, and the peak extent of MP positively correlated with the induction of hypodiploid DNA-containing cells. Our data suggest acentriolar spindle pole amplification as a hitherto undescribed activity of some declustering drugs, resulting in spindle MP in cells that may not have amplified centrosomes. In general, declustering drugs were more toxic to cancer cell lines than non-transformed ones, with some exceptions. Through a comprehensive description and quantitative analysis of numerous phenotypes induced by declustering drugs, we propose a novel framework for the assessment of putative centrosome declustering drugs and describe cellular characteristics that may enhance susceptibility to them.

Cell Death and Disease (2014) 5, e1204; doi:10.1038/cddis.2014.164; published online 1 May 2014

Subject Category: Cancer

Solid and hematological cancers often exhibit numerical centrosome amplification (CA), the presence of more than one centrosome before $S$ phase or two thereafter, a rarity in normal human tissues. ${ }^{1} \mathrm{CA}$ correlates positively with malignancy, suggesting that CA benefits cancer cells. Indeed, CA induced tumor formation and metastasis in a Drosophila larval brain transplantation assay. ${ }^{2}$ Moreover, CA may promote metastasis by enhancing directional migration and invasion. ${ }^{3}$ Nonetheless, CA also poses a liability to cancer cells. CA may result in potentially lethal mitotic spindle MP. MP can induce death following prolonged mitotic arrest (MA) or cause multipolar mitosis, resulting in daughter cells sustaining an intolerable degree of aneuploidy. ${ }^{4,5}$ In order to escape these calamitous fates, cancer cells employ centrosome clustering mechanisms to assemble a pseudo-bipolar mitotic spindle. ${ }^{6-8}$ Centrosome clustering not only circumvents death but also promotes low-grade chromosome missegregation, which may drive tumor evolution. ${ }^{9,10}$

Various factors are critical for centrosome clustering, such as cortical actin, the spindle assembly checkpoint (SAC), cell adhesion and polarity regulators, the Ndc80 microtubule- kinetochore attachment complex, augmin complex members, and microtubule motors (e.g., dynein and HSET). ${ }^{11-14}$ Inhibition of the kinesin-14 HSET has attracted attention lately because it causes death selectively in cancer cells with supernumerary centrosomes. ${ }^{11}$ Based on these promising findings, two HSET inhibitors have been developed recently. ${ }^{15,16}$ Inhibitors of centrosome clustering are selective for cancer cells because most healthy adult human cells do not exhibit CA, precluding their dependence on clustering mechanisms. ${ }^{6,17}$ Consequently, putative centrosome declustering agents have emerged as promising anticancer drugs. These agents include griseofulvin (GF), noscapine (Nos), and Nos derivatives (e.g., brominated noscapine (BN) and reduced $B N(R B N))$, all of which modulate microtubule dynamicity, as well as the phenanthrene-derived poly(ADPribose) polymerase inhibitor, PJ-34 (PJ).

Like traditional spindle poisons, declustering drugs are known to cause G2/M phase arrest (Nos, ${ }^{18} \mathrm{BN},{ }^{18} \mathrm{RBN},{ }^{19}$ $\left.\mathrm{GF}^{20} \mathrm{PJ}^{21}\right)$. Spindle poisons induce MA by perturbing microtubule attachment to kinetochores, thereby activating the SAC. ${ }^{22}$ The SAC prevents cyclin B1 degradation, resulting

\footnotetext{
${ }^{1}$ Department of Biology, Georgia State University, Atlanta, GA, USA; ${ }^{2}$ Department of Mathematics and Statistics, Georgia State University, Atlanta, GA, USA and ${ }^{3}$ Neuroscience Institute, Georgia State University, Atlanta, GA, USA

*Corresponding author: R Aneja, Department of Biology, Georgia State University, 614 PSC, 100 Piedmont Avenue, Atlanta, GA 30303, USA. Tel: +1 404 413 5417; Fax: +1 404413 5300; Email: raneja @ gsu.edu

Keywords: centrosome declustering; centrosome amplification; microtubule-targeting agents; mitotic arrest; spindle multipolarity; spindle pole amplification Abbreviations: CA, centrosome amplification; MP, multipolarity; MA, mitotic arrest; SAC, spindle assembly checkpoint; Nos, noscapine; GF, griseofulvin; BN, brominated noscapine; RBN, reduced BN; PJ, PJ-34

Received 19.8.13; revised 26.1.13; accepted 07.2.14; Edited by E Baehrecke
} 
in sustained $\mathrm{MA}^{23}$ The fates of spindle poison-treated, mitotically arrested cells are thought to be governed by two competing pathways: one that induces apoptosis in mitosis (via caspase-dependent mechanisms) and another that induces mitotic exit (via progressive degradation of cyclin B1). ${ }^{22,24}$ Mitotic exit may culminate in apoptosis in the subsequent interphase or may permit cell survival. ${ }^{25}$ Nevertheless, controversy surrounds the role of MA in inducing apoptosis. Specifically, it remains unclear which factors - the extent, onset, and/or duration of MA - are critical for inducing apoptosis. 22,25

Despite their common ability to induce MA, declustering agents differ in their clinical indications and molecular targets. GF is an antifungal and Nos is an antitussive, both with different binding sites on microtubules, whereas $\mathrm{PJ}$ is a phenanthrene-derived poly(ADP-ribose) polymerase inhibitor with no known microtubule-binding capacity. To date, no study has quantitatively compared the declustering abilities of these diverse agents, which may contrast given their pharmacologic differences. Furthermore, these agents paradoxically are effective in cancer lines, such as HeLa, PC3, and DLD-1 cells, which lack significant $\mathrm{CA}^{26}$ (e.g., Nos and $\mathrm{BN},{ }^{18} \mathrm{GF},{ }^{20,27} \mathrm{PJ},{ }^{28}$ and $\mathrm{RBN}^{19}$ ). Unless these drugs also induce $\mathrm{CA}$ (such as occurs with $\mathrm{BN}^{29}$ and $R B N^{19}$ ), MP is perhaps being induced in such cell lines in a centrosome declustering-independent manner. It was recently demonstrated that inhibition of HSET can induce MP via acentrosomal pole formation in cancer cells irrespective of centrosome number. ${ }^{30}$ Thus, 'acentrosomal pole amplification' represents a potential mechanism by which declustering drugs might induce spindle MP in cancer cells without $\mathrm{CA}$.

Herein, we seek to quantitatively determine which of these phenotypes - MA, CA, declustering, acentrosomal pole amplification, and/or spindle MP - promote cancer cell death. This information can provide a conceptual framework to aid rational design of novel centrosome declustering drugs.

\section{Results}

Characterization of MA induced by centrosome declustering drugs. To evaluate the impact of putative declustering drugs on cell cycle progression and hypodiploidy ( $<2 \mathrm{~N}$ DNA content, which may indicate apoptotic cells), MDA-MB-231 (231), PC3, and HeLa cells were treated with different concentrations of declustering drugs, stained with propidium iodide, labeled with anti-MPM2 antibody, and then assessed by flow cytometry at multiple time points over $48 \mathrm{~h}$. The chosen cell lines displayed different levels of endogenous CA. 231 cells (mutant p53) exhibit high levels of CA $(\sim 20-45 \%)^{11,31}$ compared with PC3 (p53 null) and HeLa (wild-type but E6-inactivated p53), which have low basal levels of CA. Consistent with previous reports, our data showed that all drugs induced sustained MA (at least $2 \times$ mitotic cells compared with untreated control cultures) at the concentrations indicated. The duration, highest degree, and rapidity of onset of MA varied between drugs, drug concentrations, and cell lines (Figures 1a and b). In general, the maximum MA achieved was less pronounced in Nos- and PJ-treated cells (Figures 1a and b). Drug-induced onset of
MA was corroborated by substantial increases in cyclin B1 levels in all cell lines (Figure 1c). For most cases, prolonged MA ( $\sim 24 \mathrm{~h}$ in duration) was followed by a substantial increase in the subG1 population fraction (Figures 1a and b). In all cases, we observed significant increases in cleaved caspase-3 over controls (Figure 1c), suggesting apoptosis. Instances wherein the subG1 fraction was elevated without cleaved caspase-3 may either represent caspase-independent cell death or the presence of hypodiploid cells whose fate is unclear. In general, we found no consistent associations between the extent, duration, or timing of MA within drugs or across cell lines (detailed in the Supplementary Text, Supplementary Figure 1, and Supplementary Tables 1 and 2). Altogether, although centrosome declustering drugs induced MA, significant differences existed in the (i) extents and durations of MA, (ii) the size of the subG1 population, (iii) the rapidity of the onset of MA and hypodiploidy, and (iv) the extent to which hypodiploidy was accompanied by caspase-dependent apoptosis (Figures 1a-c) even within a given cell line.

Declustering drugs induce $\mathrm{CA}$ in cancer cell lines. As RBN increases the expression of Plk4, ${ }^{19}$ a mediator of CA, we investigated whether other declustering drugs affect the expression of PLK4 along with two other mediators of CA, Cyclin E and Aurora A. Interestingly, all the drugs we studied increased PLK4, Cyclin E, and Aurora A compared with untreated cultures (Figure 2a). Consequently, we assessed $\mathrm{CA}$ in cultures treated with different concentrations of declustering drugs for $6,12,18$, or $24 \mathrm{~h}$ and untreated controls via microscopy. Centrosomes were identified by $\gamma$-tubulin and centrin-2 colocalization at discrete foci. Interestingly, all drugs tested induced CA in a statistically significant manner in at least one cell type and drug concentration (10 or $25 \mu \mathrm{M}$ for all drugs except GF, which was used at 25 and $50 \mu \mathrm{M}$ ). The average percentages of CA over $24 \mathrm{~h}$ and the associated fold increases over controls are shown in Figure 3 and Supplementary Figure 2, respectively. The peak percent CA detected over $24 \mathrm{~h}$ is shown in Supplementary Figure $4 a$ (only statistically significant $(P<0.05)$ increases over control values are represented in the figures, and a more detailed analysis is described in the Supplementary Data). Representative confocal micrographs of CA in interphase and mitotic cells, both control and drug treated, are depicted in Figure 4. We did not find significant correlations between the degree of CA (Figure 3a) and the expression levels of PLK4, Cyclin E, and Aurora A (Figure 2). Although declustering drugs might induce CA through pathways independent of these proteins, we conjecture that the levels of these proteins required to induce CA may simply vary between cell lines. Furthermore, many of the cells in which CA was induced may have died, precluding their quantitation. To determine whether the CA-inducing activity of declustering drugs is restricted to cancer cells, we treated two non-malignant cell lines, mammary fibrocystic (MCF10A) cells and adult human dermal fibroblasts with these drugs. We found that RBN, GF, and PJ did induce CA (for details, see Supplementary Text, Supplementary Figures 3 and 4) and cell death (Supplementary Table 3) in these cell lines to varying extents. In summary, all the centrosome declustering 
a

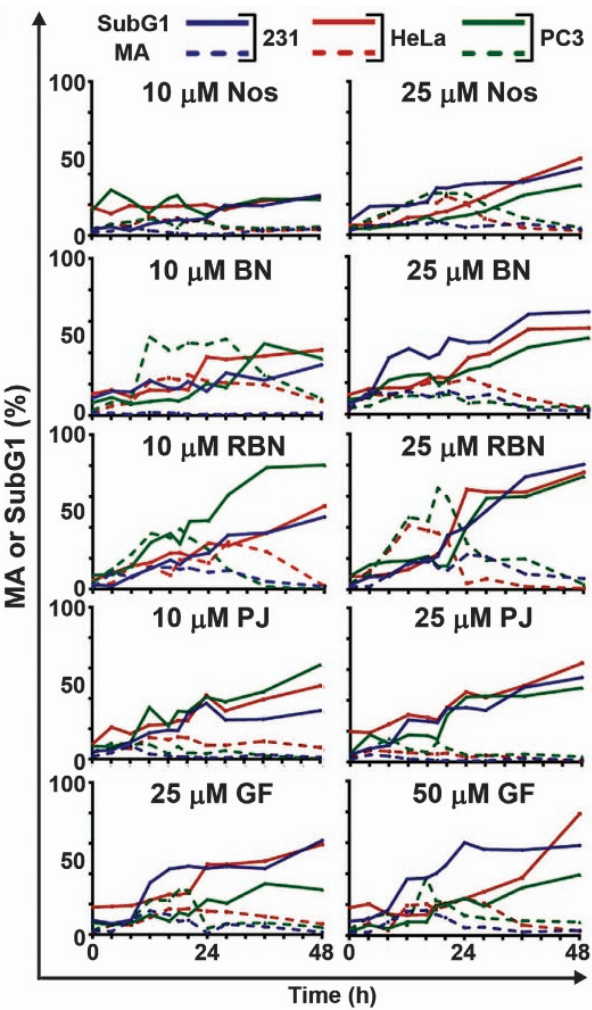

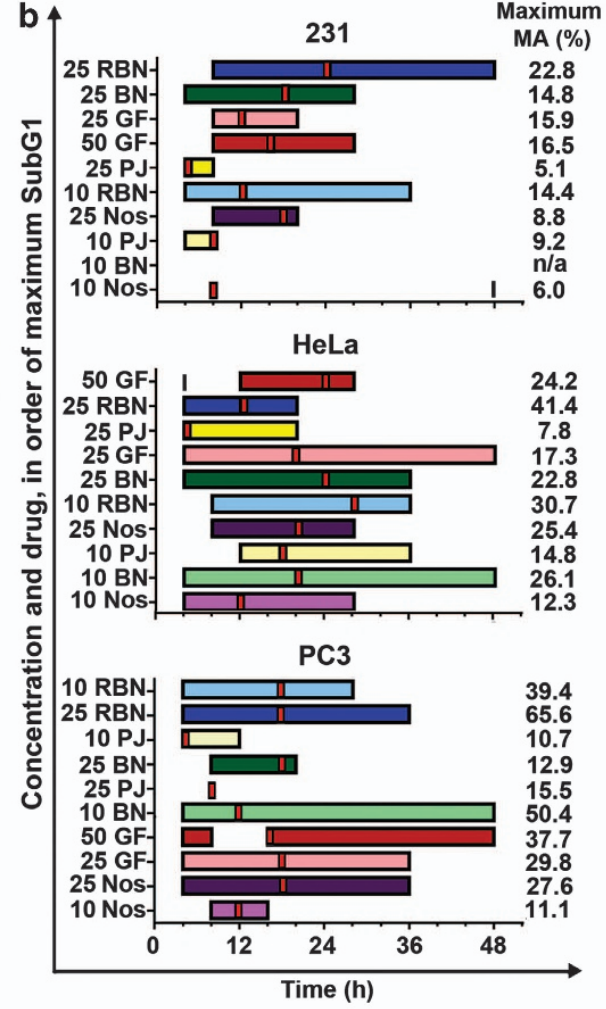

HeLa

PC3

C

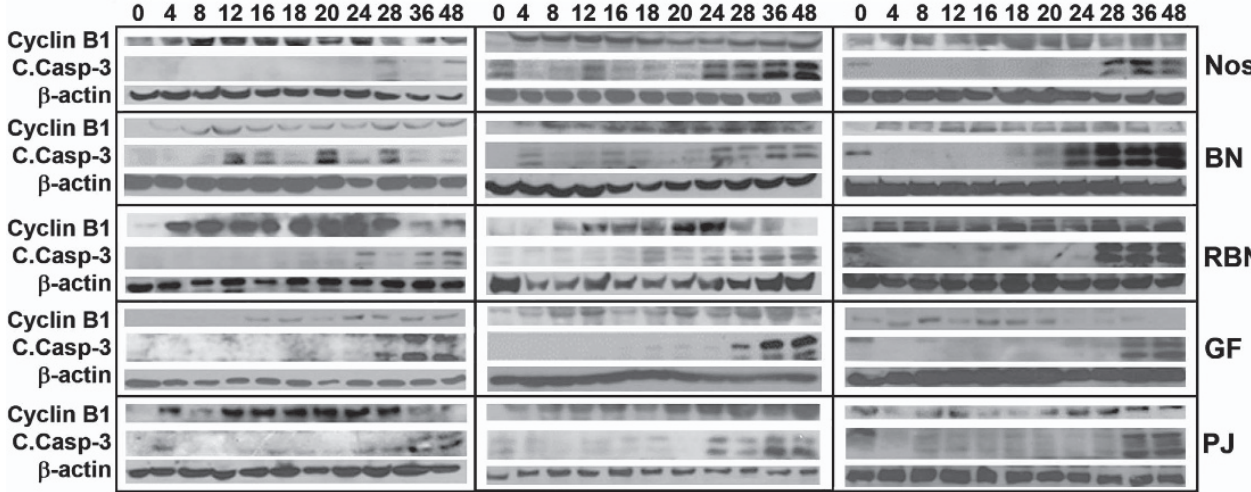

Figure 1 Mitotic arrest (MA) phenotypes observed upon treatment with putative centrosome declustering drugs. (a) SubG1 and mitotically arrested cell population fractions with respect to time post-treatment with various putative declustering drugs. Declustering drugs included Nos, BN, RBN, PJ, and GF, all at 10 and $25 \mu \mathrm{M}$ except GF, which was used at 25 and $50 \mu \mathrm{M}$, and cell lines included 231, PC3, and HeLa. These cell lines demonstrated differential susceptibility to various agents depending on drug concentration over the $48 \mathrm{~h}$ time period. In general, MA increased from $0 \mathrm{~h}$ to a peak near $24 \mathrm{~h}$, followed by a decline in MA that coincided with increases in subG1 fractions. Results are representative of three independent experiments. (b) Duration of MA and peak MA by maximum subG1 fraction. Drugs are ranked in order of increasing peak subG1 from bottom to top along the yaxis. The duration of MA (defined as the duration for which the mitotic population in drug-treated cells was greater than two times that in control cells) is plotted along the $x$ axis. The time at which peak MA occurred is illustrated as a red bar and the value of peak MA is listed to the right of the graph. In 231 cells, 10 BN did not cause any MA; therefore, no bar is plotted. For 10 Nos in 231 cells and 25 PJ in PC3 cells, MA was observed at only one time point and is depicted using a single red bar. Some drugs produced a MA that then subsided and ultimately recurred, resulting in two bars being plotted, namely 50 GF in HeLa and PC3 cells. (c) Western blotting of cell cycle-related proteins and caspase- 3 , a marker for apoptosis. To assess cell cycle progression following treatment with different declustering drugs (all at $25 \mu \mathrm{M}$ ), cell lysates were obtained at multiple time points over $48 \mathrm{~h}$ and immunoblotted for Cyclins $\mathrm{E}$ and B1. Increased levels of both cyclins compared with controls ( $0 \mathrm{~h}$ ) were detected across cell lines with variable expression patterns depending on the drug and cell line. To evaluate apoptosis, cleaved caspase-3 (C. Caspase-3) was immunoblotted and eventual increases over controls were universally detected, typically by $24 \mathrm{~h}$

drugs in our study were also centrosome-amplifying drugs, depending on the cell line and drug concentration.

Effect of putative declustering agents on centrosome declustering and spindle MP. Having identified that all the declustering drugs in our study induce CA, we were interested to quantitatively evaluate the extent to which they induce MP and declustering. MP was considered low grade if there were only 3 or 4 spindle poles and high grade if there were $\geq 5$ poles. We found that all declustering drugs, at one 
231

$0481216182024283648 h \quad 0481216182024283648$ h 04481216182024283648 h

Aurora

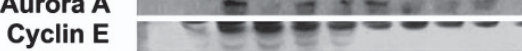

B-actin

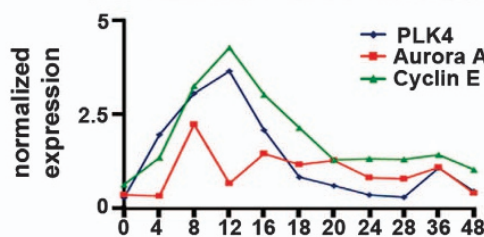

PLK4

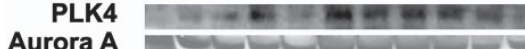

Cyclin E

p-actin

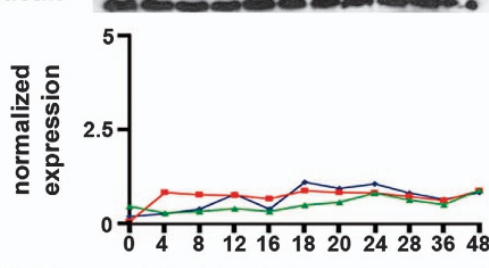

PLK4

Aurora A

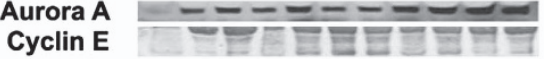

$\beta$-actin

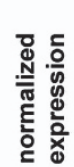
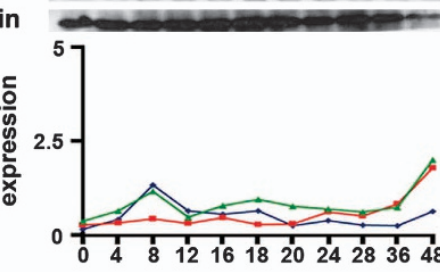

PLK4
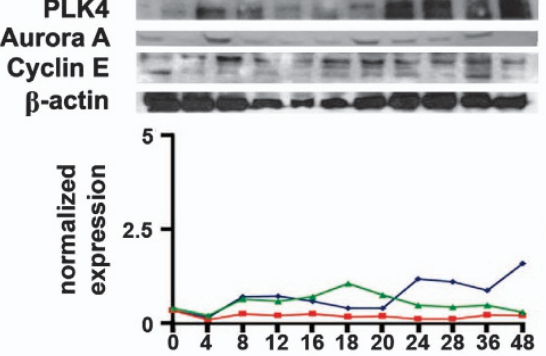

PLK4

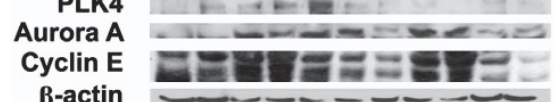

B-actin

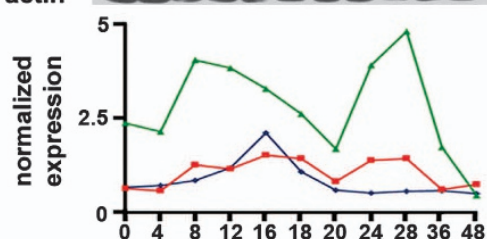

HeLa

PC3
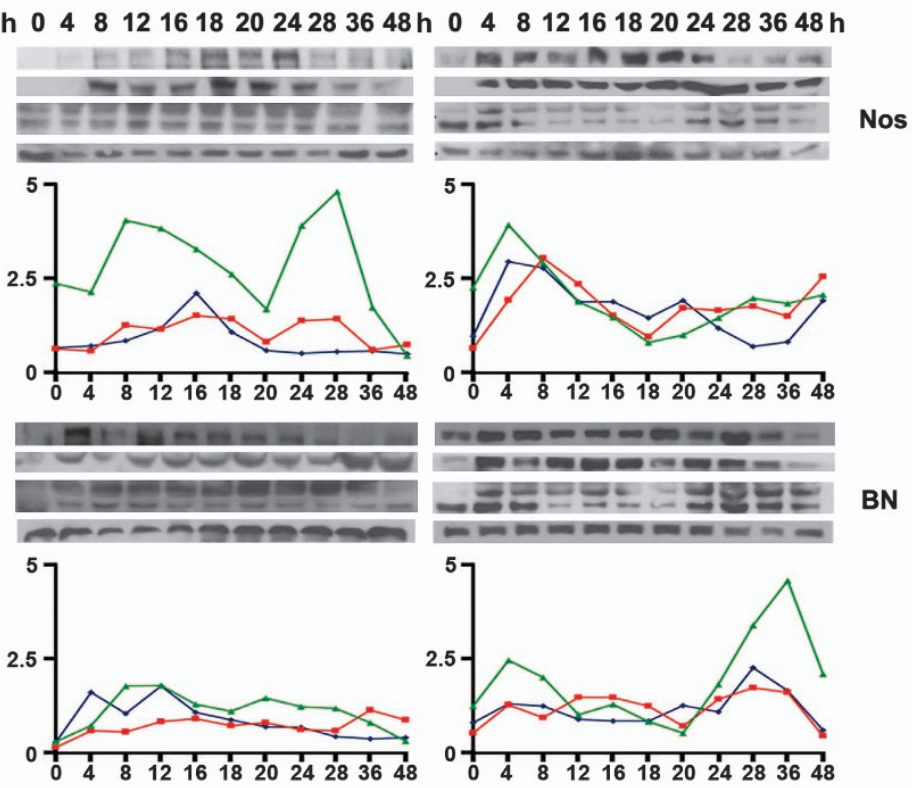

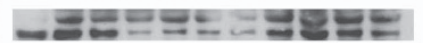

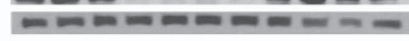

BN

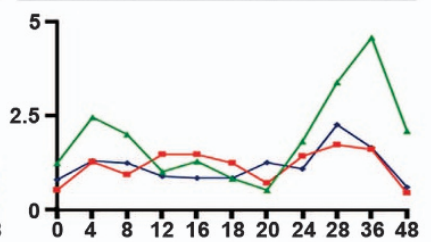

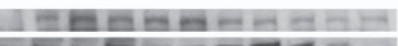
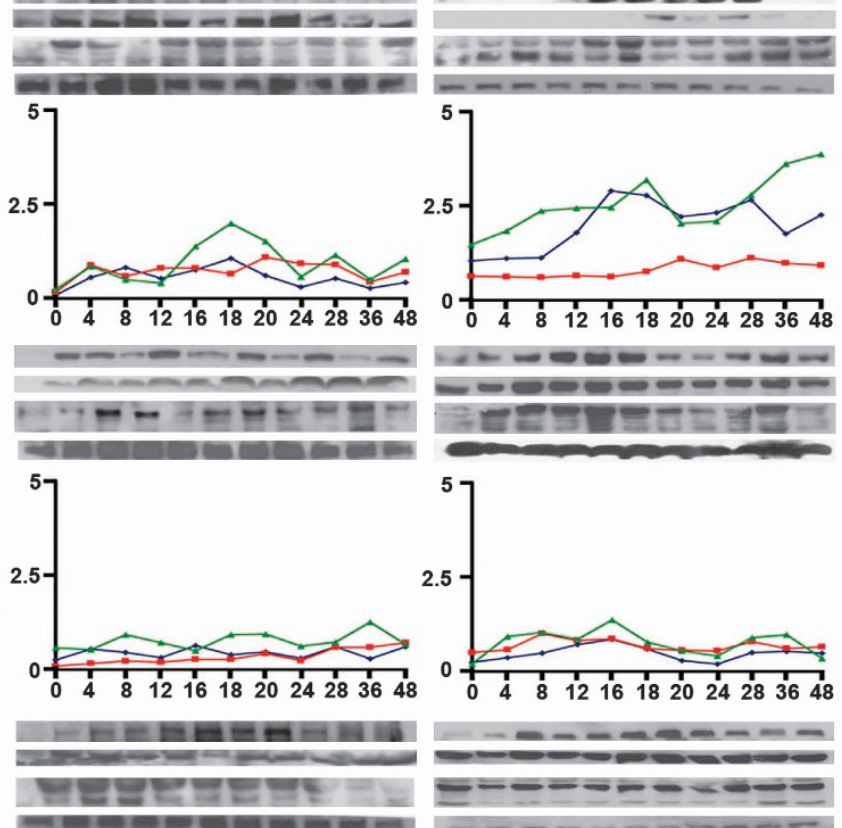

PJ
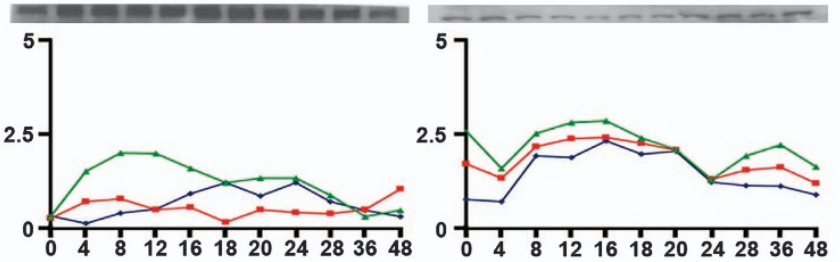

Figure 2 Centrosome declustering drug-induced changes in expression levels of markers of centrosome amplification. To evaluate the levels of some of the well-established markers of CA upon treatment with declustering drugs at a concentration of $25 \mu \mathrm{M}$, the levels of PLK4, Cyclin E, and Aurora A were assessed by western blotting, revealing eventual increases over untreated controls across cell lines. Increase in expression levels of PLK4 and Aurora A was generally rapid, often appearing by $4 \mathrm{~h}$. Levels tended to vary thereafter depending on the drug and cell line. Densitometry was performed to quantitate the changes in levels of CA markers relative to $\beta$-actin over time, and the changes in actin-normalized expression levels over the time-course of the experiment are depicted graphically beneath each sets of blots. As the Cyclin $\mathrm{E}$ blots revealed two closely placed bands ( 49 and $43 \mathrm{kDa}$ ) corresponding to the two spliced forms, the Cyclin $\mathrm{E}$ band intensity was generated as a sum of the two band intensities 


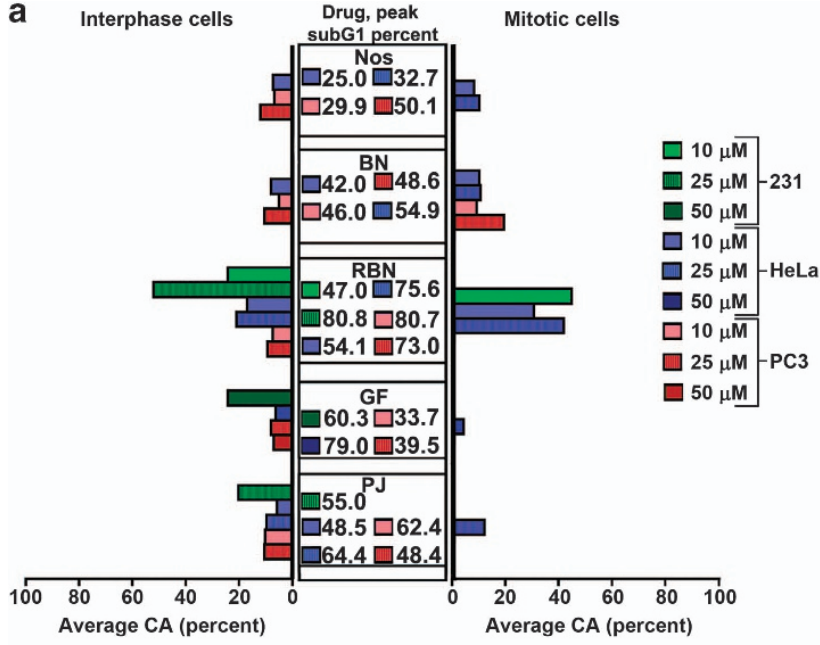

b

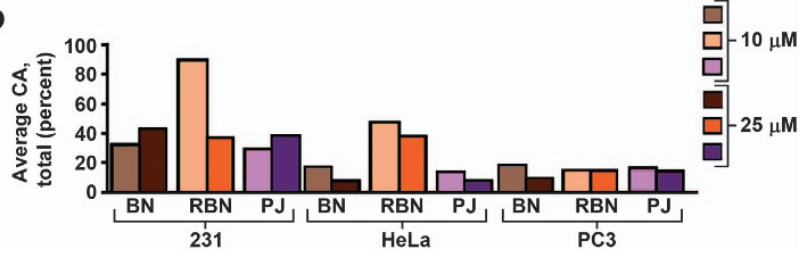

Figure 3 Average CA observed over $24 \mathrm{~h}$ and its relationship with peak subG1 for each drug treatment regimen. (a) Displayed are only statistically significant $(P<0.05)$ increases in average $C A$ over controls. To calculate average $C A$, the sum of percentage of (interphase or mitotic) cells showing CA at the $6,12,18$, and $24 \mathrm{~h}$ time points was divided by 4. (b) Depiction of the sum of average CA (interphase plus mitotic) observed when 231 cells were treated with RBN, BN, and PJ, compared with the treatment of HeLa and PC3 cells with the same three drugs

or both concentrations, induced spindle MP in at least one cell type above control levels (Figure 5a). Several of the drugs induced acentrosomal or 'acentriolar' poles (wherein at least one spindle pole stained positively for $\gamma$-tubulin but not centrin-2; Figure 5a), a phenotype not previously reported for these particular drugs. This phenotype has been reported following knockdown of HSET. ${ }^{30}$ We found that acentriolar poles were more readily induced in HeLa than in PC3 or 231 cells (Figure 5a). The mechanism undergirding this phenotype is presently unknown. Some of the forces that tether together supernumerary centrosomes may also preserve spindle pole integrity, ${ }^{8}$ and our observations support that hypothesis.

Next, we evaluated the extents to which these drugs induced declustering. We found that the extent of total declustering (the percentage of cells with amplified centrosomes in which no centrosomes were clustered) induced by all the drug regimens was the lowest in 231 cells, which have higher endogenous CA (Figure 5b). By contrast, in HeLa and PC3 cells, which have comparatively low levels of CA, a majority of the amplified centrosomes were found to be totally declustered (Figure 5b). For comparison, we assessed druginduced MP, declustering, and acentrosomal pole formation in non-malignant cell lines (for details, see Supplementary Text and Data in Supplementary Figure 5a). We found that RBN, GF, and PJ significantly induced MP over control levels, and the supernumerary centrosomes induced tended be declustered.
Ultimately, it appears that the drugs tested largely induce spindle MP in a declustering-independent manner, at least in the cancer cell lines tested here. Declustering drugs may therefore prove effective in cancers regardless of the extent of CA present.

Cross talk between drug-induced spindle MP, declustering, and drug efficacy. Next, we probed the associations between drug-induced spindle MP, centrosome declustering, and drug efficacy (subG1 extent) in order to identify the phenotypes that contributed most to cell death. We used beta regression (a statistical methodology more appropriate for proportions data than linear regression when very low or high percentages are observed) to analyze correlates of peak subG1. For this technique, pseudo $R^{2}$ (the squared correlation of linear predictor and link-transformed response) is reported rather than $R^{2}$ as in linear regression, and it indicates the goodness-of-fit of the model.

We discovered that across all drugs and cell lines, peak MP significantly correlated with peak subG1 $(P=0.00840$, pseudo $R^{2}=0.321$ ), suggesting that generation of spindle MP is a shared mechanism whereby declustering drugs trigger cell death. Importantly, we found no significant associations between CA and spindle MP, which corroborates our finding that declustering drugs appear to induce spindle MP by disrupting spindle pole and/or centrosome integrity, which in some cases may also decluster centrosomes if an excess is present. Within 231 cells, we found an even stronger, positive correlation with a very good fit between peak high-grade MP and peak subG1 (Figure 6ai; $P=0.006$; pseudo $R^{2}=0.833$ ), underscoring that a desirable attribute for declustering drugs is the ability to induce high-grade rather than low-grade MP. We also found that a model including both peak high-grade and low-grade MP together was better in predicting peak subG1 $\left(P=0.001 ;\right.$ pseudo $\left.R^{2}=0.860\right)$ (Figure 6aii). Specifically, within this model, the prediction of peak subG1 using peak high-grade MP was very highly statistically significant $(P<0.00001)$ and the beta coefficient was positive, indicating a positive correlation between peak high-grade MP and subG1 generation. The prediction of peak subG1 using peak low-grade MP was very highly statistically significant $(P=0.00001)$, and the beta coefficient was negative, indicating a negative correlation between peak low-grade MP and peak subG1. This finding is consistent with the notion that high-grade MP engenders intolerably severe aneuploidy that is likely to culminate in cell death, whereas low-grade MP is more likely to be survivable and perhaps advantageous to cancer cells. Clear trends were not uncovered for centrosome declustering and subG1 across drugs, although we cannot rule out its importance within individual drugs, as the number of data points for peak subG1 was limiting.

In HeLa cells, peak MP (any grade) positively correlated with peak subG1 ( $P=0.0055$; pseudo $R^{2}=0.575$; Figure $\left.6 \mathrm{ci}\right)$. Also, peak high-grade MP positively correlated somewhat with peak subG1 ( $P=0.028$; pseudo $R^{2}=0.271$; Figure 6 cii). Notably, the peak acentriolar pole percentage positively correlated with peak subG1 $\left(P=0.0023\right.$; pseudo $R^{2}=0.600$; Figure 6ciii), so daughter HeLa cells without centrosomes may be inviable. Indeed, based on the pseudo $R^{2}$ value, peak 


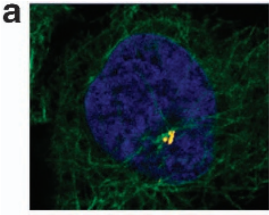

Control, 231

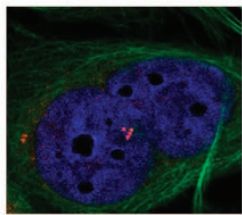

BN, PC3

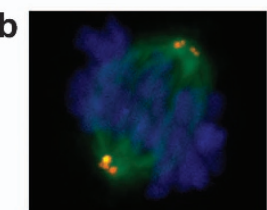

Control, 231

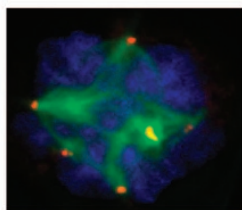

BN, PC3

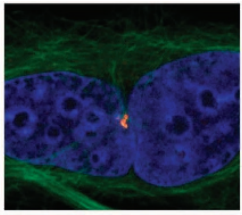

Control, 231

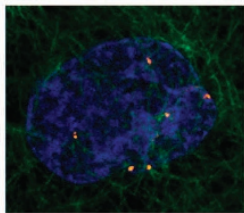

RBN, PC3
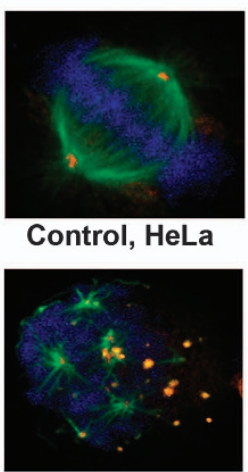

RBN, HeLa

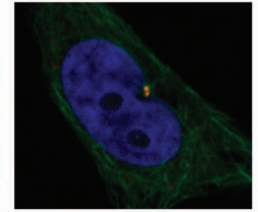

Control, HeLa

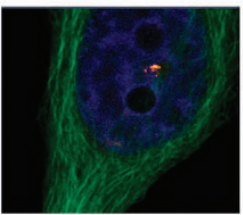

RBN, 231

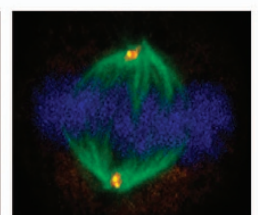

Control, PC3

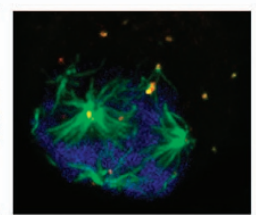

RBN, 231

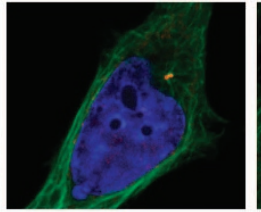

Control, PC3

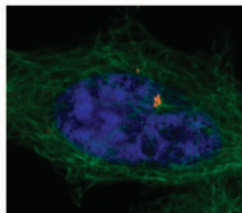

GF, HeLa

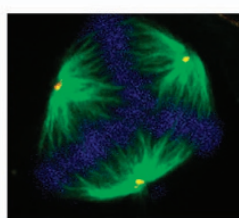

Nos, HeLa

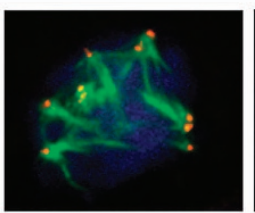

GF, HeLa

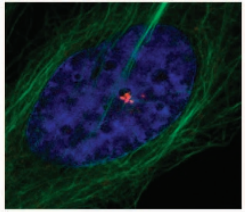

Nos, HeLa

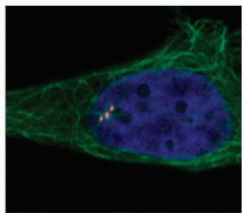

PJ, PC3

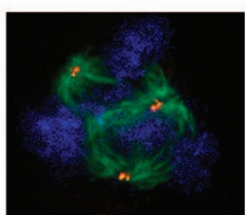

BN, HeLa

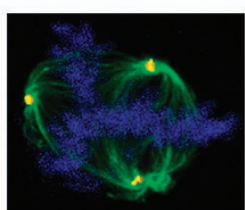

PJ, HeLa

Figure 4 Representative confocal micrographs depicting centrosome amplification in controls and drug-treated cancer cells. (a) Interphase and (b) mitotic cells. Only displayed are drug/cell line combinations in which statistically significant $(P<0.05)$ fold increases in CA were found in mitotic cells, although all controls are shown regardless of the extent of endogenous CA. Blue $=\mathrm{DNA}$, green $=$ microtubules, red $=\gamma$-tubulin, and orange $=$ centrin-2

acentriolar pole formation was superior to all other variables in predicting peak subG1. Peak total declustering also positively correlated with peak subG1 $\left(P=0.020\right.$; pseudo $R^{2}=0.424$; Figure 6civ), strengthening the idea that more extensive declustering kills more cancer cells.

In PC3 cells, we did not find an association between peak MP and peak subG1 across drugs. However, when we analyzed the correlation between the average fold increase in CA induction with peak subG1 percent, we found an interesting trend. In PC3 cells, the proportion variable (peak subG1) always lay within the $30-70 \%$ range and the other variable (fold increase in CA) was continuous, so we implemented linear regression for analysis. We found that the average fold increase in CA in interphase positively correlated with peak subG1 $\left(P=0.057 ; R^{2}=0.619\right.$; Figure $\left.6 \mathrm{~b}\right)$, suggesting that an increase in CA may promote cell death.

We also analyzed the impact of treatment with declustering drugs on spindle MP and subG1 induction in non-transformed cells (for details, see Supplementary Data and Supplementary Figure 6). In both MCF10A cells and human dermal fibroblasts, peak MP positively correlated with peak subG1 ( $R^{2}=0.82$ with $P=0.003$ and $R^{2}=0.89$ with $P<0.001$, respectively), suggesting that MP is also toxic to normal cells.

\section{Discussion}

Declustering of supernumerary centrosomes is a promising chemotherapeutic approach that has recently come to light.
Centrosome declustering kills cancer cells with supernumerary centrosomes while sparing normal cells, which rarely have supernumerary centrosomes. To date, drugs that decluster centrosomes have been grouped together given that they share this ability; however, the precise mechanisms by which they decluster centrosomes may differ and are unknown. It is also unclear whether centrosome declustering is their sole, or even primary, cellular activity. Ours is the first study to assess the differential abilities of various declustering drugs to induce MA, CA, MP, declustering, acentrosomal pole amplification, and cell death in different malignant and non-malignant cell lines. It would be valuable to have a framework to quantitatively evaluate novel declustering drugs to improve their efficacy via rational design. Our study lays the foundation for defining such a framework, which could guide development of a next generation of declustering drugs that are even more effective in killing cancer cells and even less toxic to normal cells.

Centrosome declustering drugs induce MA followed by death, ${ }^{19,27,28,32,33}$ and the duration of MA induced by some microtubule-targeting drugs determines cell death after mitotic exit. ${ }^{34}$ In concordance, we found that declustering drugs induce MA, which is followed by a peak in the subG1 fraction. However, we also found great variability in the extent of MA, how rapidly its onset occurs, its duration, and the extent of hypodiploidy generated between drugs and cell lines. Differences were also apparent in the extent to which MA was accompanied by the appearance of cleaved caspase-3, an early marker of apoptosis. There were no clear associations 

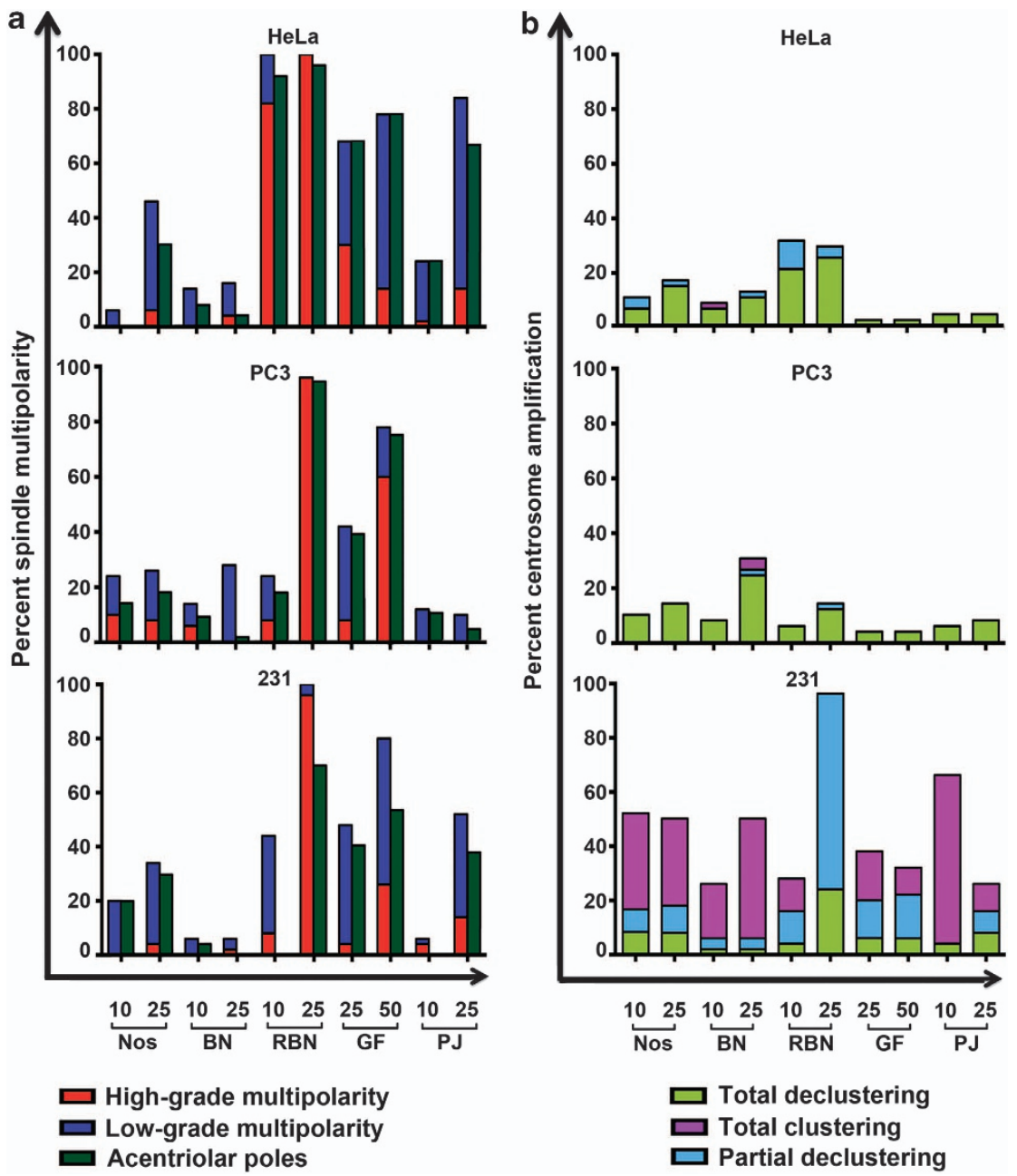

Figure 5 (a) Peak MP and peak acentriolar pole formation induced by different declustering drugs in 231, HeLa, and PC3 cells. The maximum extents of MP induction of high grades ( $5+$ poles) and low grades (3-4 poles) and acentriolar pole formation (at least one pole without centrioles) across a 24-h period are given for all drugs. (b) Peak CA and declustering of amplified centrosomes induced in 231, HeLa, and PC3 cells. The maximum extent of CA in mitosis over $24 \mathrm{~h}$ is depicted by the height of the bar. The extent of total clustering (all centrosomes clustered at two poles), total declustering (all centrosomes separated to different poles), and partial declustering (one or more poles with $2+$ centrosomes) are given for that same time point

between peak MA, the duration of MA, and the timing of the onset of MA with hypodiploidy or the various centrosome- and spindle-related phenotypes we observed. These findings convey the complexity of the cellular responses to these drugs and suggest that the mechanisms of actions of these drugs probably differ significantly.

A central finding of our study is that all the declustering drugs upregulate PLK4, Cyclin E, and Aurora A and induce CA (Figure 2). This finding helps to clarify the seeming contradiction that centrosome declustering drugs are effective in cell lines without much CA. Although it has been previously shown that $\mathrm{BN}^{29}$ and $\mathrm{RBN}^{19}$ induce $\mathrm{CA}$, no other studies to our knowledge have demonstrated induction of CA by Nos, GF, or PJ. It currently does not appear that the CA induced by these drugs is coupled in any way to their declustering activity, although further studies are needed to establish conclusively whether these two activities are truly independent. Another key finding from our study is that declustering drugs can trigger 'spindle pole amplification' via two means, acting separately or in concert: (i) genesis of acentriolar poles and (ii) amplification and declustering of centrosomes, both of which precipitate spindle MP.

SubG1 fraction (i.e., cell death) typically exceeded the extent of CA, often by a wide margin (Figures 1 and 2, Supplementary Table 3). Thus, CA and declustering alone cannot explain the efficacy of these agents in the cell lines examined. Many reports have demonstrated that MP can be induced without centrosome declustering, such as through spindle pole fragmentation, loss of microtubule anchoring at the centrosome, PCM fragmentation, or centriole separation in mitosis. ${ }^{35-38}$ Consequently, it is conceivable that 'declustering drugs' are inducing MP in a centrosome declusteringindependent manner, such as by 'declustering' (defocusing) spindle poles. For instance, treatment of HeLa cells with $50 \mu \mathrm{M}$ GF $\left(\mathrm{IC}_{50}=35 \mu \mathrm{M}^{27}\right)$ for $24 \mathrm{~h}$ induced MP in $72 \%$ of cells, even though we found that $\leq 4 \%$ of untreated controls had $\mathrm{CA}$, as others have found. ${ }^{26}$ We also discovered that $50 \mu \mathrm{M}$ GF induced only a small increase in the extent of CA in HeLa cells. Although the minority of multipolar cells with CA did exhibit centrosome declustering in the presence of GF, the 


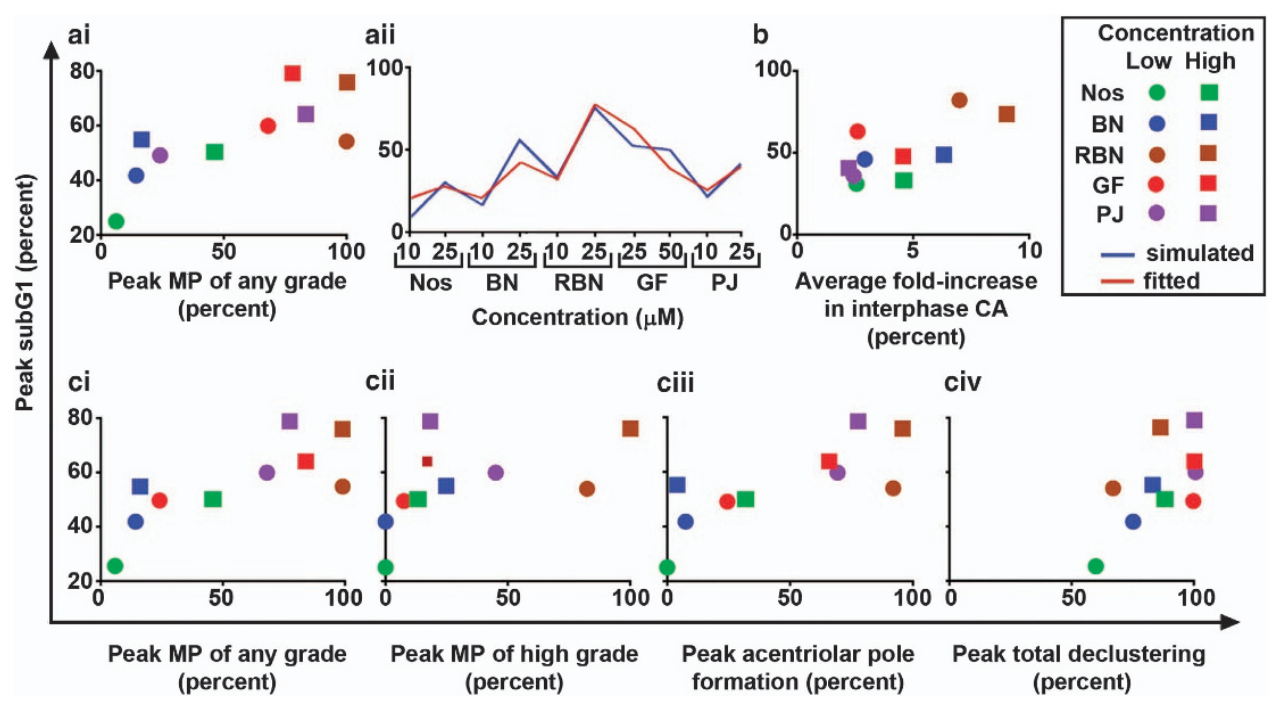

Figure 6 Correlates of peak subG1 percent within cell lines. (a) 231 cells, beta regression. (ai) In 231 cells, a clear trend was found for increasing peak MP of any grade and peak subG1, which was highly statistically significant $\left(P=0.006\right.$; pseudo $R^{2}=0.833$ ). (aii) Furthermore, multiple regression using peak MP (high grade) and peak MP (low grade) produced an even better, statistically significant fit (red line) compared with simulated values $\left(P=0.001\right.$; pseudo $\left.R^{2}=0.860\right)$. Within this model, both variables were very highly statistically significant $(P<0.0001)$, with peak high-grade MP showing a positive correlation and peak low-grade MP showing a negative correlation with peak subG1 (based on the sign of the beta coefficients). (b) PC3 cells, linear regression. In these cells, the average fold increase in interphase CA shows some association with peak subG1, which almost reached statistical significance and which produced a good fit $\left(P=0.057 ; R^{2}=0.619\right)$. (c) HeLa cells, beta regression. (ci) Increasing peak MP of any grade was associated with peak subG1 $\left(P=0.0055\right.$; pseudo $\left.R^{2}=0.575\right)$, as was (cii). Increasing peak MP of high grade $\left(P=0.028 ;\right.$ pseudo $\left.R^{2}=0.271\right)$. (ciii) Increasing peak acentriolar pole formation $\left(P=0.0023\right.$; pseudo $\left.R^{2}=0.600\right)$, and (civ) peak total declustering $\left(P=0.020\right.$; pseudo $\left.R^{2}=0.424\right)$

majority of multipolar cells exhibited acentrosomal poles without centrosome declustering, as they did not have extra centrosomes that could be declustered. Nos, likewise, is effective in cells without much endogenous $\mathrm{CA}$, such as HeLa $\left(\mathrm{IC}_{50}=25 \mu \mathrm{M}^{32}\right)$, and Nos frequently induced MP by generating acentrosomal poles (Figure 5a). Nos also induced further CA in HeLa and PC3 cells and achieved nearly complete declustering of these amplified centrosomes. Intriguingly, although we confirmed that $\mathrm{BN}$ induced $\mathrm{CA}$ that closely paralleled MP across cell lines (i.e., cells with CA were generally the ones that were also MP; Figure 5a), we discovered that the subG1 population exceeded the percentage of cells with CA and/or MP. This finding suggests that additional mechanisms may contribute to this drug's anticancer activity, even though CA and declustering appear to operate as well. We also verified that RBN substantially increases CA across cell lines but it often produces MP spindles with one or more acentrosomal poles. Consequently, like all the other putative centrosome declustering agents that we studied, it does not seem that RBN is a 'pure' declustering drug but may additionally disrupt spindle pole integrity or otherwise cause spindle pole amplification, a downstream consequence of which is centrosome declustering. Altogether, it seems that MP itself, however it is generated (whether via bona fide centrosome declustering or acentrosomal spindle pole amplification), is a key driver of cell death in 231 and HeLa cells, although CA may instead be mechanistically involved in cell death in PC3 cells.

Ultimately, the diversity of previously unrecognized or under-appreciated phenotypes uncovered by our study, illustrated in Figure 7, raises a host of questions that represent ample opportunities for future investigations. We have discerned certain phenotypes that contribute to cell death,

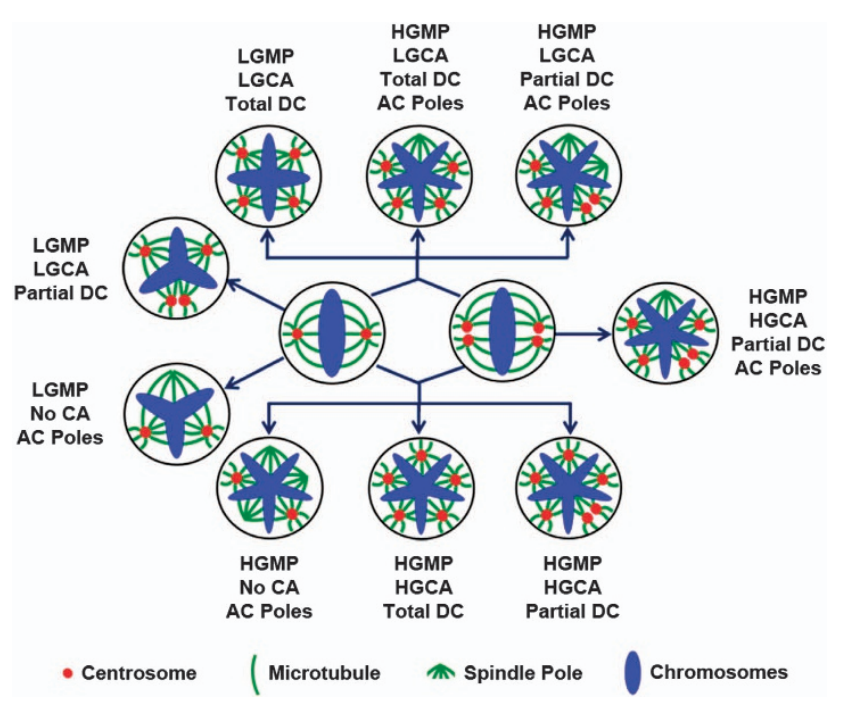

Figure 7 Diversity of phenotypes produced by putative centrosome declustering drugs. One of the two central cells with bipolar spindles show normal centrosome number (left cell) and the other one shows amplified centrosomes (right cell). Both types of cells are susceptible to the declustering agents. For the cell without CA, acentriolar pole formation (i.e., pole amplification or pole declustering) and MP (low- or high-grade) may be induced. Alternatively, these agents may induce CA and permit bona fide centrosome declustering to occur, partially or in total, with or without acentriolar pole formation. For the cell with CA, genuine centrosome declustering may occur, partially or in total, with or without acentriolar pole formation, and with or without further CA. AC, acentriolar; DC, declustering; HGCA, high-grade CA; HGMP, high-grade MP; LGCA, low-grade CA; LGMP, low-grade MP

which may guide rational development of these drugs or formulation of synergistic drug combinations to enhance chemotherapeutic efficacy. Altogether, our data highlight the potential utility of centrosome declustering drugs in combatting 
malignancy given that we found cancer cells are often more susceptible to these agents than normal cells. Interestingly, we uncovered that centrosome declustering drugs may act partially or primarily through spindle pole amplification. Declustering may either operate in addition to spindle pole amplification or may simply be a corollary of that process. Future studies are awaited to further unravel the precise mechanisms by which currently available declustering drugs operate to guide rational design of novel members of this promising class.

\section{Materials and Methods}

Cell lines, cell culture, and drugs. HeLa, MDA-MB-231 (231), and PC3 cells were grown in medium (Dulbecco's modified Eagle's medium for HeLa and 231 cells, Roswell Park Memorial Institute medium for PC-3 cells; Invitrogen, Carlsbad, CA, USA) supplemented with $10 \%$ HyClone fetal bovine serum (Thermo Scientific, Waltham, MA, USA) and maintained in a $95 \% \mathrm{CO}_{2}$ atmosphere at $37^{\circ} \mathrm{C}$. BN and RBN were prepared as described previously. ${ }^{19,39}$ Nos, GF, and PJ were purchased from Sigma (St. Louis, MO, USA). Cells were grown to $70 \%$ confluence and treated with drug or vehicle ( $0.1 \%$ dimethyl sulfoxide) followed by processing for flow cytometry, immunofluorescence confocal microscopy, or immunoblotting as previously described. ${ }^{29}$

Immunofluorescence microscopy and western blotting. Primary antibodies against $\gamma$-tubulin, $\alpha$-tubulin, and centrin-2 (Abcam, Cambridge, MA USA), Alexa 488-, 555-, and 647-conjugated secondary antibodies (Invitrogen), and DAPI (Invitrogen) were diluted in $2 \% \mathrm{BSA} / 1 \times$ PBS 1:2000. Confocal microscopy was performed using the Zeiss LSC 700 microscope (Oberkochen, Germany) with a 1.4 NA oil-immersion lens, and image processing was performed with Zen software (Oberkochen, Germany). For each drug at a particular concentration $(10,25$, or $50 \mu \mathrm{M})$ in a specific cell line (HeLa, 231 , or PC3 cells), 200 cells in randomly selected fields were assessed for each time point $(0,12,18$, and $24 \mathrm{~h}$ ). If the number of mitotic cells out of 200 did not reach $n=50$, additional randomly selected mitotic cells were assessed until $n=50$. The number of interphase cells was never less than $n=133$. Mitotic index was calculated for each time point, and for both interphase and mitotic cells, the number of $\gamma$-tubulin/ centrin-positive foci (i.e., centrosomes) in each cell was recorded. For mitotic cells, the number of spindle poles was also recorded, along with the number of centrosomes present, if any, at each pole. Western blotting was performed as previously described ${ }^{40}$ using lysates from cells treated with $25 \mu \mathrm{M}$ drug for $0,4,8$, $12,16,18,20,24,28,36$, or $48 \mathrm{~h}$.

Cell cycle analysis. The flow cytometric evaluation of cell cycle status was done using FlowJo software (Ashland, OR, USA). After the treatment, cells were harvested at different time intervals, washed twice with ice-cold PBS, and fixed in $70 \%$ ethanol for at least $24 \mathrm{~h}$. Cell pellets were then washed with PBS and added with $0.5 \mathrm{ml}$ of RNase A (2 mg/ml) and stained with MPM-2 primary mouse antibody followed by Alexa-488 secondary antibody. In addition, $0.5 \mathrm{ml}$ of propidium iodide $(0.1 \%$ with $0.6 \%$ Triton X-100 in PBS) was added for $45 \mathrm{~min}$ in the dark followed by analysis on a FACS Cantor flow cytometer (BD Canto, Franklin Lakes, NJ, USA). Experiments were repeated in triplicate.

Statistical analysis. To compare proportions between samples, Fisher's exact test was performed using SPSS. Sample size was always $n \geq 50$. The only exception occurred with calculation of the proportion of cells exhibiting centrosome declustering (the percent of cells with declustered centrosomes out of all cells with CA at each time point), as the number of cells with CA was typically $<50$. In all cases, results were considered significant if $P<0.05$. To perform regression using proportions, beta regression was used as described by Ferrari and Cribari-Neto ${ }^{41}$ using MATLAB.

\section{Conflict of Interest}

The authors declare no conflict of interest.

1. Chan JY. A clinical overview of centrosome amplification in human cancers. Int J Biol Sci 2011; 7: 1122-1144

2. Basto R, Brunk K, Vinadogrova T, Peel N, Franz A, Khodjakov A et al. Centrosome amplification can initiate tumorigenesis in flies. Cell 2008; 133: 1032-1042.
3. Ogden A, Rida PC, Aneja R. Heading off with the herd: how cancer cells might maneuver supernumerary centrosomes for directional migration. Cancer Metastasis Rev 2012; 32: 269-287.

4. Vitale I, Galluzzi L, Castedo M, Kroemer G. Mitotic catastrophe: a mechanism for avoiding genomic instability. Nat Rev Mol Cell Biol 2011; 12: 385-392.

5. Holland AJ, Cleveland DW. Boveri revisited: chromosomal instability, aneuploidy and tumorigenesis. Nat Rev Mol Cell Biol 2009; 10: 478-487.

6. Kramer A, Maier B, Bartek J. Centrosome clustering and chromosomal (in)stability: a matter of life and death. Mol Oncol 2011; 5: 324-335.

7. Ogden A, Rida PC, Aneja R. Let's huddle to prevent a muddle: centrosome declustering as an attractive anticancer strategy. Cell Death Differ 2012; 19: 1255-1267.

8. Godinho SA, Kwon M, Pellman D. Centrosomes and cancer: how cancer cells divide with too many centrosomes. Cancer Metastasis Rev 2009; 28: 85-98.

9. Silkworth WT, Nardi IK, Scholl LM, Cimini D. Multipolar spindle pole coalescence is a major source of kinetochore mis-attachment and chromosome mis-segregation in cancer cells. PLoS One 2009; 4: e6564

10. Ganem NJ, Godinho SA, Pellman D. A mechanism linking extra centrosomes to chromosomal instability. Nature 2009; 460: 278-282.

11. Kwon M, Godinho SA, Chandhok NS, Ganem NJ, Azioune A, Thery M et al. Mechanisms to suppress multipolar divisions in cancer cells with extra centrosomes. Genes Dev 2008; 22: 2189-2203.

12. Leber B, Maier B, Fuchs $F$, Chi J, Riffel $P$, Anderhub $S$ et al. Proteins required for centrosome clustering in cancer cells. Sci Transl Med 2010; 2: 33ra38.

13. Quintyne NJ, Reing JE, Hoffelder DR, Gollin SM, Saunders WS. Spindle multipolarity is prevented by centrosomal clustering. Science 2005; 307: 127-129.

14. Fielding AB, Lim S, Montgomery K, Dobreva I, Dedhar S. A critical role of integrin-linked kinase, ch-TOG and TACC3 in centrosome clustering in cancer cells. Oncogene 2011; 30: 521-534.

15. Wu J, Mikule K, Wang W, Su N, Petteruti P, Gharahdaghi $F$ et al. Discovery and mechanistic study of a small molecule inhibitor for motor protein KIFC1. ACS Chem Biol 2013; 8: 2201-2208.

16. Watts CA, Richards FM, Bender A, Bond PJ, Korb O, Kern O et al. Design, synthesis, and biological evaluation of an allosteric inhibitor of HSET that targets cancer cells with supernumerary centrosomes. Chem Biol 2013; 20: 1399-1410.

17. Korzeniewski N, Hohenfellner M, Duensing S. The centrosome as potential target for cancer therapy and prevention. Expert Opin Ther Targets 2013; 17: 43-52.

18. Aneja R, Vangapandu SN, Lopus M, Viswesarappa VG, Dhiman N, Verma A et al. Synthesis of microtubule-interfering halogenated noscapine analogs that perturb mitosis in cancer cells followed by cell death. Biochem Pharmacol 2006; 72: 415-426.

19. Pannu V, Rida PC, Ogden A, Clewley R, Cheng A, Karna P et al. Induction of robust de novo centrosome amplification, high-grade spindle multipolarity and metaphase catastrophe: a novel chemotherapeutic approach. Cell Death Dis 2012; 3: e346.

20. Rebacz B, Larsen TO, Clausen MH, Ronnest MH, Loffler H, Ho AD et al. Identification of griseofulvin as an inhibitor of centrosomal clustering in a phenotype-based screen. Cancer Res 2007: 67: 6342-6350.

21. Inbar-Rozensal D, Castiel A, Visochek L, Castel D, Dantzer F, Izraeli S et al. A selective eradication of human nonhereditary breast cancer cells by phenanthridine-derived polyADP-ribose polymerase inhibitors. Breast Cancer Res 2009; 11: R78.

22. Matson DR, Stukenberg PT. Spindle poisons and cell fate: a tale of two pathways. $\mathrm{Mol}$ Interv 2011; 11: 141-150.

23. Sakurikar N, Eichhorn JM, Chambers TC. Cyclin-dependent kinase-1 (Cdk1)/cyclin B1 dictates cell fate after mitotic arrest via phosphoregulation of antiapoptotic $\mathrm{Bcl}-2$ proteins. J Biol Chem 2012; 287: 39193-39204.

24. Gascoigne KE, Taylor SS. Cancer cells display profound intra- and interline variation following prolonged exposure to antimitotic drugs. Cancer Cell 2008; 14: 111-122.

25. Manchado E, Guillamot M, Malumbres M. Killing cells by targeting mitosis. Cell Death Differ 2012; 19: 369-377

26. Starita LM, Machida $Y$, Sankaran S, Elias JE, Griffin $K$, Schlegel BP et al. BRCA1-dependent ubiquitination of gamma-tubulin regulates centrosome number. Mol Cell Biol 2004; 24: 8457-8466.

27. Rathinasamy K, Jindal B, Asthana J, Singh P, Balaji PV, Panda D. Griseofulvin stabilizes microtubule dynamics, activates p53 and inhibits the proliferation of MCF-7 cells synergistically with vinblastine. BMC Cancer 2010; 10: 213.

28. Castiel A, Visochek L, Mittelman L, Dantzer F, Izraeli S, Cohen-Armon M. A phenanthrene derived PARP inhibitor is an extra-centrosomes de-clustering agent exclusively eradicating human cancer cells. BMC Cancer 2011; 11: 412

29. Karna P, Rida PC, Pannu V, Gupta KK, Dalton WB, Joshi H et al. A novel microtubulemodulating noscapinoid triggers apoptosis by inducing spindle multipolarity via centrosome amplification and declustering. Cell Death Differ 2011; 18: 632-644.

30. Kleylein-Sohn J, Pollinger B, Ohmer M, Hofmann F, Nigg EA, Hemmings BA et al. Acentrosomal spindle organization renders cancer cells dependent on the kinesin HSET. J Cell Sci 2012; 125(Pt 22): 5391-5402.

31. D'Assoro AB, Busby R, Suino K, Delva E, Almodovar-Mercado GJ, Johnson $\mathrm{H}$ et al. Genotoxic stress leads to centrosome amplification in breast cancer cell lines that have an inactive G1/S cell cycle checkpoint. Oncogene 2004; 23: 4068-4075.

32. Ye K, Ke Y, Keshava N, Shanks J, Kapp JA, Tekmal RR et al. Opium alkaloid noscapine is an antitumor agent that arrests metaphase and induces apoptosis in dividing cells. Proc Natl Acad Sci USA 1998; 95: 1601-1606. 
33. Jaiswal AS, Aneja R, Connors SK, Joshi HC, Multani AS, Pathak S et al. 9-bromonoscapine-induced mitotic arrest of cigarette smoke condensate-transformed breast epithelial cells. J Cell Biochem 2009; 106: 1146-1156.

34. Bekier ME, Fischbach R, Lee J, Taylor WR. Length of mitotic arrest induced by microtubule-stabilizing drugs determines cell death after mitotic exit. Mol Cancer Ther 2009; 8: 1646-1654.

35. Logarinho E, Maffini S, Barisic M, Marques A, Toso A, Meraldi P et al. CLASPs prevent irreversible multipolarity by ensuring spindle-pole resistance to traction forces during chromosome alignment. Nat Cell Biol 2012; 14: 295-303.

36. Gergely F, Draviam VM, Raff JW. The ch-TOG/XMAP215 protein is essential for spindle pole organization in human somatic cells. Genes Dev 2003; 17: 336-341.

37. Wu Q, He R, Zhou H, Yu AC, Zhang B, Teng J et al. Cep57, a NEDD1-binding pericentriolar material component, is essential for spindle pole integrity. Cell Res 2012; 22: 1390-1401.

38. Torres JZ, Summers MK, Peterson D, Brauer MJ, Lee J, Senese $S$ et al. The STARD9/Kif16a kinesin associates with mitotic microtubules and regulates spindle pole assembly. Cell 2011; 147: 1309-1323.

39. Zhou J, Gupta K, Aggarwal S, Aneja R, Chandra R, Panda D et al. Brominated derivatives of noscapine are potent microtubule-interfering agents that perturb mitosis and inhibit cell proliferation. Mol Pharmacol 2003; 63: 799-807.
40. Karna P, Zughaier S, Pannu V, Simmons R, Narayan S, Aneja R. Induction of reactive oxygen species-mediated autophagy by a novel microtubule-modulating agent. J Biol Chem 2010; 285: 18737-18748.

41. Ferrari S, Cribari-Neto F. Beta regression for modelling rates and proportions. J Appl Stat 2004; 31: 799-815.

cc)(1)(2) Cell Death and Disease is an open-access journal published by Nature Publishing Group. This work is licensed under a Creative Commons Attribution-NonCommercialShareAlike 3.0 Unported License. The images or other third party material in this article are included in the article's Creative Commons license, unless indicated otherwise in the credit line; if the material is not included under the Creative Commons license, users will need to obtain permission from the license holder to reproduce the material. To view a copy of this license, visit http://creativecommons.org/ licenses/by-nc-sa/3.0/

Supplementary Information accompanies this paper on Cell Death and Disease website (http://www.nature.com/cddis) 